\title{
Design and Simulation of Measurement-Based Correlation Models for Shadow Fading
}

\author{
Matthias Pätzold ${ }^{1}$, Nurilla Avazovi ${ }^{1}$, Van Duc Nguyen ${ }^{2}$ \\ ${ }^{1}$ Faculty of Engineering and Science, University of Agder, Grimstad, Norway \\ ${ }^{2}$ Faculty of Electronics and Telecommunications, Hanoi University of Science and Technology, Hanoi, \\ Vietnam
}

Correspondence: Matthias Pätzold, matthias.paetzold@uia.no

Manuscript communication: received 18 May 2011, accepted 15 June 2011

\begin{abstract}
This paper deals with the design of measurement-based correlation models for shadow fading. Based on the correlation model, we design a simulation model using the sum-of-sinusoids (SOS) method to enable the simulation of spatial lognormal processes characterizing real-world shadow fading scenarios. The model parameters of the simulation model are computed by applying the $L_{p}$-norm method (LPNM). This method facilitates an excellent fitting of the simulation model's autocorrelation function (ACF) to that of measured channels. Our study includes an evaluation of all important statistical quantities of the proposed measurement-based simulation model, such as the probability density function (PDF), spatial ACF, decorrelation and coherence distance, as well as the level-crossing rate (LCR) and the average duration of fades (ADF). A comparison with the Gudmundson correlation model shows that the developed measurement-based correlation model outperforms the former one by far in terms of the goodness of fit to the measured data. The proposed measurementbased simulation model allows to study the effects of long-term fading on the performance of mobile communication systems under real-world shadow fading conditions.
\end{abstract}

Keywords- Shadow fading, lognormal process, sum-of-sinusoids, autocorrelation function, level-crossing rate, average duration of fades.

\section{INTRODUCTION}

In wireless communications, shadowing causes longterm variations in the received signal. This effect is attributed to the fluctuation of the envelope due to large objects obstructing the propagation paths between the base station (BS) and the mobile station (MS). The objects may include mountains, buildings, trees, and cars. Based on experimental studies [1-4], it is generally accepted that the signal fluctuations caused by shadowing can be modelled by a lognormal process. To account for the shadowing effects in land mobile terrestrial channels, the lognormal process has been incorporated in the Suzuki model [5], the Loo model [6], the modified Loo model [7], and the extended Loo model [8]. Lognormal processes play also an important role in a variety of other areas in wireless communications. In [9] and [10], e.g., velocity estimation schemes based on the spatial correlation properties have been proposed. In [11], Yamamoto et al. studied the impact of the shadowing correlation on the coverage of multi-hop cellular systems. Moreover, the lognormal process plays a key role in the study of the quality of service (QoS) and the performance analysis of handover procedures in wireless systems $[12,13]$.

The spatial correlation properties of lognormal processes have been investigated in [14-17]. In [14], a simple analytical model for the ACF of the lognormal process was proposed by Gudmundson. He came up with a negative exponential correlation model, which has been improved later in [15]. The drawback of the Gudmundson model is that the LCR of the lognormal process is infinite. Despite this drawback, the Gudmundson correlation model has been adopted in the past by many researchers for lack of alternatives. Meanwhile, heuristic alternative correlation models can be found, e.g., in [17], where the Gaussian and the Butterworth models have been proposed.

Recent works on empirical correlation models to characterize shadowing processes in different areas have been published in [18-21]. In [21], Zhang et al. proposed a novel spatial correlation model for shadow fading in urban macro environments. The proposed model is based on empirical results obtained from a wideband radio channel measurement campaign at 2.35 $\mathrm{GHz}$ in an urban area. The authors showed that the proposed model provides a good match to the empirical results and outperforms the double exponential model.

In contrast to our related work in [16, 17], where the focus was on fundamental aspects as well as on the modelling of empirical and heuristic correlation models, we present in this paper a design method for measurement-based spatial correlation models to enable the simulation of real-world shadowing processes. The model parameters are computed by using the LPNM, which allows a quasi-perfect fitting of the 
spatial ACF of the shadowing simulator to that of measured channels. The concept of SOS has been applied to design the shadowing simulator. Analytical expressions are provided for the PDF, ACF, LCR, ADF, as well as for the decorrelation and coherence distance. The design concept is applied to measurement data collected in suburban and urban areas. The results show that the statistics of the proposed correlation model fit very well to those of the measured channel.

The rest of this paper is organized as follows. Section 2 reviews spatial lognormal processes. In Section 3, the shadowing simulator is derived by using the SOS principle. We also present briefly all relevant statistical properties of the shadowing simulator. In Section 4, we show how the LPNM can be applied to compute the model parameters. The evaluation of the main statistical properties of the simulation model is the topic of Section 5. In Section 6, we present some simulation results of the ergodic spatial shadowing processes. Finally, Section 7 provides the conclusion of the paper.

\section{Review of Lognormal Processes}

In the spatial domain, the shadowing effects are usually modelled by a spatial lognormal process $\lambda(x)$, which can be expressed as

$$
\lambda(x)=10^{\left[\sigma_{L} \mu(x)+m_{L}\right] / 20}
$$

where $\mu(x)$ is a real-valued zero-mean Gaussian process with unit variance. The symbols $\sigma_{L}$ and $m_{L}$ stand for the shadow standard deviation and the area mean, respectively. The shadow standard deviation $\sigma_{L}$ is an area specific constant, and the area mean $m_{L}$ is determined by the path loss between the BS and the MS.

The PDF $p_{\lambda}(y)$ of $\lambda(x)$ is known as the lognormal distribution

$$
p_{\lambda}(y)=\frac{20}{\sqrt{2 \pi} \ln (10) \sigma_{L} y} e^{-\frac{\left(20 \log _{10}(y)-m_{L}\right)^{2}}{2 \sigma_{L}^{2}}}, \quad y \geq 0 .
$$

The mean $m_{\lambda}$ and the variance $\sigma_{\lambda}^{2}$ of the spatial lognormal process $\lambda(x)$ in (1) can be expressed as

$$
\begin{gathered}
m_{\lambda}=e^{m_{0}+\sigma_{0}^{2} / 2}, \\
\sigma_{\lambda}^{2}=e^{2 m_{0}+\sigma_{0}^{2}}\left(e^{\sigma_{0}^{2}}-1\right)
\end{gathered}
$$

respectively, where $m_{0}=m_{L} \ln (10) / 20$ and $\sigma_{0}=$ $\sigma_{L} \ln (10) / 20$.

The Gaussian process $\mu(x)$ is characterized by the spatial ACF $r_{\mu \mu}(\Delta x)$, which is defined as $r_{\mu \mu}(\Delta x)=$ $E\{\mu(x) \mu(x+\Delta x)\}$, where $\Delta x:=x_{2}-x_{1}$ denotes the spatial separation.

In [16], it is shown that the spatial ACF $r_{\lambda \lambda}(\Delta x)$ of $\lambda(x)$, which is defined as $r_{\lambda \lambda}(\Delta x)=E\{\lambda(x) \lambda(x+\Delta x)\}$, can be expressed in terms of $r_{\mu \mu}(\Delta x)$ as follows

$$
r_{\lambda \lambda}(\Delta x)=e^{2 m_{0}+\sigma_{0}^{2}\left[1+r_{\mu \mu}(\Delta x)\right]} \text {. }
$$

The mean power of the spatial lognormal process $\lambda(x)$ equals the spatial ACF $r_{\lambda \lambda}(\Delta x)$ at the origin, i.e., $E\left\{\lambda^{2}(x)\right\}=r_{\lambda \lambda}(0)=e^{2\left(m_{0}+\sigma_{0}^{2}\right)}$.
The autocovariance function (ACVF) $r_{\lambda \lambda}^{c}(\Delta x)$ of the spatial lognormal process $\lambda(x)$ is defined as $r_{\lambda \lambda}^{c}(\Delta x)=$ $r_{\lambda \lambda}(\Delta x)-m_{\lambda}^{2}$. By using (3a) and (4), this function can be written as

$$
r_{\lambda \lambda}^{c}(\Delta x)=e^{2 m_{0}+\sigma_{0}^{2}}\left[e^{\sigma_{0}^{2} r_{\mu \mu}(\Delta x)}-1\right] .
$$

The ACVF $r_{\lambda \lambda}^{c}(\Delta x)$ is useful to determine the coherence distance of the spatial lognormal process. The coherence distance $D_{c}$ is a measure for the distance between $x_{2}$ and $x_{1}$, i.e., $D_{c}=x_{2}-x_{1}$, beyond which the random variates $\lambda\left(x_{1}\right)$ and $\lambda\left(x_{2}\right)$ can be considered as statistically uncorrelated. This quantity is defined as the minimum distance, which fulfills the following equation

$$
r_{\lambda \lambda}^{c}\left(D_{c}\right)=\frac{r_{\lambda \lambda}^{c}(0)}{2}
$$

By substituting (5) in (6), we can alternatively compute $D_{c}$ by solving

$$
r_{\mu \mu}\left(D_{c}\right)-\frac{1}{\sigma_{0}^{2}} \ln \left(\frac{e^{\sigma_{0}^{2}}+1}{2}\right)=0 .
$$

If the spatial ACF $r_{\mu \mu}\left(D_{c}\right)$ has an inverse (see Section 5.3), then $D_{c}$ can be expressed in closed form. Otherwise, (7) has to be solved numerically by using root finding algorithms.

Other important characteristic quantities of the spatial lognormal process $\lambda(x)$ are the LCR $N_{\lambda}(r)$ and the ADF $T_{\lambda_{-}}(r)$. The LCR $N_{\lambda}(r)$ describes how often the spatial lognormal process $\lambda(x)$ crosses a given level $r$ from up to down (or from down to up) per unit of length. In [16], it has been shown that the LCR $N_{\lambda}(r)$ of lognormal processes $\lambda(x)$ can be expressed as

$$
N_{\lambda}(r)=\frac{\sqrt{\gamma}}{2 \pi} e^{-\frac{20 \log _{10}(r)-m_{L}{ }^{2}}{2 \sigma_{L}^{2}}}, r \geq 0
$$

where $\gamma=-\ddot{r}_{\mu \mu}(0)$. Here, $\ddot{r}_{\mu \mu}(0)$ denotes the second derivative of the spatial ACF $r_{\mu \mu}(\Delta x)$ at the origin with respect to $\Delta x$.

The ADF $T_{\lambda_{-}}(r)$ is the mean value for the length of the spatial intervals over which $\lambda(x)$ remains below a specified signal level $r$. The ADF $T_{\lambda_{-}}(r)$ is defined as [17]

$$
T_{\lambda_{-}}(r)=\frac{F_{\lambda_{-}}(r)}{N_{\lambda}(r)}
$$

where $F_{\lambda_{-}}(r)$ denotes the cumulative distribution function of the spatial lognormal process $\lambda(x)$, which is obtained as $F_{\lambda_{-}}(r)=\int_{0}^{r} p_{\lambda}(y) d y$.

\section{Simulation of Shadow Fading}

As a method to model Gaussian random processes with given spatial correlation properties, the SOS principle can be applied. In view of the development of mobile communication systems, this principle became very popular, because it enables the design of efficient and flexible mobile fading channel simulators. By analogy to (1), the stochastic simulation model for a spatial lognormal process is described by

$$
\hat{\lambda}(x)=10^{\left[\sigma_{L} \hat{\mu}(x)+m_{L}\right] / 20}
$$


where $\hat{\mu}(x)$ is called the SOS process, which has the following form

$$
\hat{\mu}(x)=\sum_{n=1}^{N} c_{n} \cos \left(2 \pi \alpha_{n} x+\theta_{n}\right) .
$$

Here, $c_{n}$ and $\alpha_{n}$ are constants representing the gains and the spatial frequencies, respectively, while the phases $\theta_{n}$ are independent, identically distributed (i.i.d.) random variables, each having a uniform distribution over the interval $(0,2 \pi]$. Since $c_{n}$ and $\alpha_{n}$ are constant parameters, but $\theta_{n}$ are random variables, it follows that the resulting shadow fading simulator described by (10) belongs to the class of ergodic channel simulators [22].

The spatial ACF $\hat{r}_{\mu \mu}(\Delta x)$ of $\hat{\mu}(x)$ is defined as $\hat{r}_{\mu \mu}(\Delta x)=E\{\hat{\mu}(x) \hat{\mu}(x+\Delta x)\}$, which can be expressed by using (11) as

$$
\hat{r}_{\mu \mu}(\Delta x)=\sum_{n=1}^{N} \frac{c_{n}^{2}}{2} \cos \left(2 \pi \alpha_{n} \Delta x\right) .
$$

In [16], it is shown that the PDF $\hat{p}_{\lambda}(x)$ of $\hat{\lambda}(x)$ is given by

$$
\begin{aligned}
\hat{p}_{\lambda}(y)= & \frac{40}{\sigma_{L} \ln 10 y} \int_{0}^{\infty}\left[\prod_{n=1}^{N} J_{0}\left(2 \pi c_{n} z\right)\right] \\
& \cdot \cos \left[\frac{2 \pi z\left(20 \log _{10} y-m_{L}\right)}{\sigma_{L}}\right] d z .
\end{aligned}
$$

Furthermore, in [16], it is also shown that a good approximation of the spatial ACF $\hat{r}_{\lambda \lambda}(\Delta x)$ of $\hat{\lambda}(x)$ can be obtained by replacing $r_{\mu \mu}(\Delta x)$ in (4) by $\hat{r}_{\mu \mu}(\Delta x)$, i.e.,

$$
\hat{r}_{\lambda \lambda}(\Delta x) \approx e^{2 m_{0}+\sigma_{0}^{2}\left[1+\hat{r}_{\mu \mu}(\Delta x)\right]}
$$

Consequently, the ACVF $\hat{r}_{\lambda \lambda}^{c}(\Delta x)$ of the shadowing simulator can be approximated as

$$
\hat{r}_{\lambda \lambda}^{c}(\Delta x) \approx e^{2 m_{0}+\sigma_{0}^{2}}\left[e^{\sigma_{0}^{2} \hat{r}_{\mu \mu}(\Delta x)}-1\right] .
$$

By analogy to (6), the coherence distance $\hat{D}_{c}$ of the stochastic process $\hat{\lambda}(x)$ is the value of $\Delta x=\hat{D}_{c}$ that solves the following equation

$$
\hat{r}_{\lambda \lambda}^{c}\left(\hat{D}_{c}\right)=\frac{\hat{r}_{\lambda \lambda}^{c}(0)}{2} .
$$

By substituting (15) in (16), we can find $\hat{D}_{c}$ by using the $\mathrm{ACF} \hat{r}_{\mu \mu}(\Delta x)$ of $\hat{\mu}(x)$ and solving

$$
\hat{r}_{\mu \mu}\left(\hat{D}_{c}\right)-\frac{1}{\sigma_{0}^{2}} \ln \left(\frac{e^{\sigma_{0}^{2}}+1}{2}\right)=0 .
$$

Finally, by substituting (12) in (17) and by applying root finding techniques, we can numerically determine the coherence distance $\hat{D}_{c}$.

By analogy to (8), the LCR $\hat{N}_{\lambda}(r)$ of the simulation model can also be approximated by substituting in (8) the quantity $\hat{\gamma}=-\ddot{\hat{r}}_{\mu \mu}(0)$ for $\gamma$, i.e.,

$$
\hat{N}_{\lambda}(r) \approx \frac{\sqrt{\hat{\gamma}}}{2 \pi} e^{-\frac{\left(20 \log _{10}(r)-m_{L}\right)^{2}}{2 \sigma_{L}^{2}}}, r \geq 0
$$

where

$$
\hat{\gamma}=-\left.\frac{d^{2}}{d \Delta x^{2}} \hat{r}_{\mu \mu}(\Delta x)\right|_{\Delta x=0}=2 \pi^{2} \sum_{n=1}^{N}\left(c_{n} \alpha_{n}\right)^{2} .
$$

Obviously, a necessary condition for a good approximation $\hat{N}_{\lambda}(r) \approx N_{\lambda}(r)$ is that the quantity $\hat{\gamma}$ is close to $\gamma$. Hence, a good design method for the model parameters $c_{n}$ and $\alpha_{n}$ has to guarantee that we may write $\hat{\gamma} \approx \gamma$. It should be mentioned for the sake of completeness that an exact solution of the LCR $\hat{N}_{\lambda}(r)$ can be found in [17].

Analogously to (9), the ADF $\hat{T}_{\lambda_{-}}(r)$ of the spatial shadowing simulation model is defined as

$$
\hat{T}_{\lambda_{-}}(r)=\frac{\hat{F}_{\lambda_{-}}(r)}{\hat{N}_{\lambda}(r)}
$$

where $\hat{N}_{\lambda}(r)$ is given by (18) and $\hat{F}_{\lambda_{-}}(r)$ describes the cumulative distribution function of $\hat{\lambda}(x)$, which can be obtained from (13) by using $\hat{F}_{\lambda_{-}}(r)=\int_{0}^{r} \hat{p}_{\lambda}(y) d y$.

\section{The $L_{p}$-NORM Method}

The objective of this section is to compute the model parameters $c_{n}$ and $\alpha_{n}$ such that the ACF $\hat{r}_{\mu \mu}(\Delta x)$ of the simulation model [see (12)] is close to the ACF $r_{\mu \mu}^{\star}(\Delta x)$ of a measured channel. To solve this parameter computation problem, we apply the LPNM, which is described in detail in [23]. The LPNM is one of the fundamental methods to compute the parameters of SOS simulation models. The aim of this method is to compute the model parameters $c_{n}$ and $\alpha_{n}$ such that the $L_{p}$-norm

$$
E_{r_{\mu \mu}}^{(p)}=\left[\frac{1}{\Delta x_{\max }} \int_{0}^{\Delta x_{\max }}\left|r_{\mu \mu}^{\star}(\Delta x)-\hat{r}_{\mu \mu}(\Delta x)\right|^{p} d(\Delta x)\right]^{1 / p}
$$

becomes a minimum, where $p=1,2, \ldots$ The quantity $\Delta x_{\max }$ denotes the upper limit of the interval $\left[0, \Delta x_{\max }\right]$ over which a good approximation $r_{\mu \mu}^{\star}(\Delta x) \approx \hat{r}_{\mu \mu}(\Delta x)$ is of interest. One of the advantages of the LPNM is that the procedure allows fitting the statistical properties of the shadowing simulator to measured data of realworld channels. Measurement results of spatial ACFs $r_{\mu \mu}^{\star}(\Delta x)$ characterizing suburban and urban areas are reported in [14]. Choosing $N=25$ and applying the LPNM with $p=2$ to these measured ACFs $r_{\mu \mu}^{\star}(\Delta x)$ results in the model parameters listed in Table I. With the obtained parameters, the proposed measurementbased correlation model in (12) is completely defined.

\section{Performance Evaluation of the Measurement-Based Channel Simulator}

This section discusses the performance evaluation of the designed measurement-based spatial shadowing simulator with respect to the analytical expressions in (12), (14), (18), and (20). A comparison has been made with the corresponding quantities of measured 
Table I

Parameters of the Measurement-Based Shadowing Simulator.

\begin{tabular}{|c||c|r||c|c|}
\hline \multicolumn{1}{|c||}{ Index } & \multicolumn{2}{c||}{ Suburban area } & \multicolumn{2}{c|}{ Urban area } \\
\multicolumn{1}{|c||}{$n$} & \multicolumn{2}{c|}{$c_{n}$} & \multicolumn{1}{c|}{$\alpha_{n}$} & \multicolumn{2}{c|}{$c_{n}$} & $\alpha_{n}$ \\
\hline \hline 1 & 0.2431 & -0.00036861 & 0.3579 & 0.02004459 \\
2 & 0.2939 & -0.00111374 & 0.3374 & 0.00874076 \\
3 & 0.3583 & -0.00084599 & 0.3314 & 0.07099983 \\
4 & 0.2676 & -0.00029556 & 0.1461 & 0.02926888 \\
5 & 0.2023 & 0.00018512 & 0.3576 & 0.02521945 \\
6 & 0.3941 & 0.00019357 & 0.1633 & 0.01321234 \\
7 & 0.3224 & 0.00012965 & 0.2347 & 0.01365564 \\
8 & 0.2704 & 0.00009131 & 0.3101 & -0.01073672 \\
9 & 0.2290 & -0.00144244 & 0.4280 & 0.00992094 \\
10 & 0.2049 & 0.00003980 & 0.3806 & 0.01245341 \\
11 & 0.3244 & -0.00041958 & 0.1762 & 0.00949120 \\
12 & 0.2747 & 0.00069153 & 0.4710 & 0.00558419 \\
13 & 0.3066 & 0.00041170 & 0.3361 & 0.01579579 \\
14 & 0.1513 & -0.01855732 & 0.4440 & 0.02795457 \\
15 & 0.3854 & 0.00000029 & 0.2701 & 0.11299331 \\
16 & 0.2880 & -0.00051154 & 0.1266 & 0.15421563 \\
17 & 0.1719 & 0.00865254 & 0.2201 & 0.08971074 \\
18 & 0.2911 & 0.00067489 & 0.3021 & 0.04895022 \\
19 & 0.3613 & -0.00007190 & 0.2036 & 0.13410393 \\
20 & 0.3067 & 0.00007934 & 0.1678 & 0.18152402 \\
21 & 0.3699 & 0.00008182 & 0.0916 & 0.10328293 \\
22 & 0.3652 & -0.00010209 & 0.1883 & 0.20076019 \\
23 & 0.0865 & 0.00495717 & 0.2081 & 0.22118688 \\
24 & 0.0267 & 0.02460851 & 0.1718 & 0.23977893 \\
25 & 0.1966 & 0.01014643 & 0.1172 & 0.62271175 \\
\hline
\end{tabular}

Table II

Parameters of the Gudmundson Model (from [14]).

\begin{tabular}{|c||c|c|c|c|}
\hline Shadowing area & $D$ & $\Delta x_{\max }$ & $\sigma_{L}$ & $m_{L}$ \\
\hline \hline Suburban & $503.9 \mathrm{~m}$ & $2500 \mathrm{~m}$ & $7.5 \mathrm{~dB}$ & 0 \\
\hline Urban & $8.3058 \mathrm{~m}$ & $40 \mathrm{~m}$ & $4.3 \mathrm{~dB}$ & 0 \\
\hline
\end{tabular}

channels, which were published in [14]. In our performance study, we have also included the Gudmundson model [14]. The key parameters of this model are listed in Table II. For reasons of simplicity, the area mean $m_{L}$ has been set to zero. The correctness of all analytical results has been confirmed by simulation. Therefore, the spatial lognormal process $\hat{\lambda}(x)$ in (12) has been simulated by using the parameters $c_{n}$ and $\alpha_{n}$ from Table I and $\sigma_{L}$ and $m_{L}$ from Table II. The phases $\theta_{n}$ have been considered as i.i.d. random variables.

\subsection{Evaluation of the PDF}

The results of the PDF $p_{\lambda}(y)$ [see (2)] of the lognormal process are shown by the solid line in Figure 1. The graphs of the density $\hat{p}_{\lambda}(y)$ [see (13)] of the shadowing simulator are represented by the dashed line, which matches perfectly the solid line. The analytical results for $\hat{p}_{\lambda}(y)$ have been obtained by substituting the model parameters listed in Table I in (13), while $\sigma_{L}$ and $m_{L}$ have been taken from Table II.

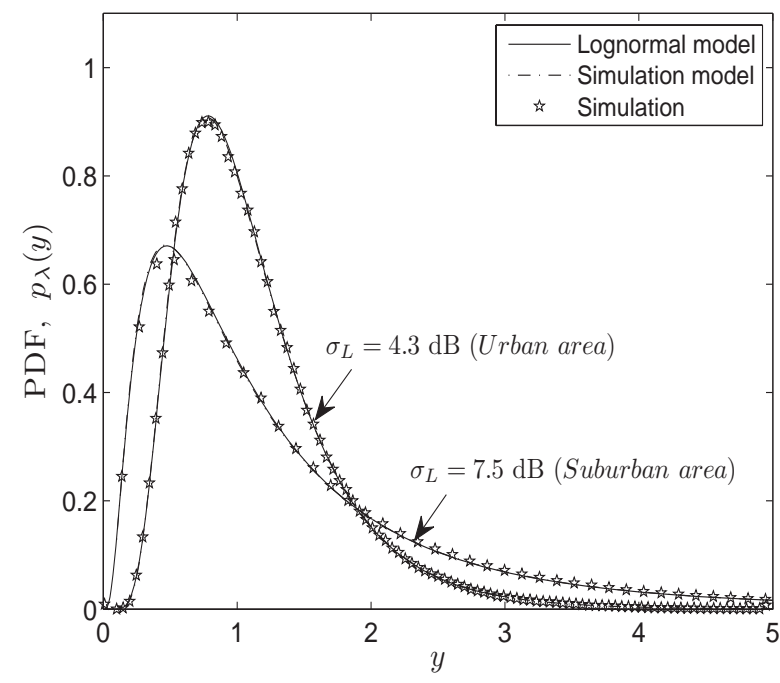

Figure 1 . The density $p_{\lambda}(y)$ of the lognormal model in comparison with the density $\hat{p}_{\lambda}(y)$ of the measurement-based simulation model for suburban and urban areas ( LPNM with $p=2$ and $N=25$ ).

\subsection{Evaluation of the Spatial ACF}

For comparison, we use the well-known Gudmundson model [14], which is described by the spatial ACF $r_{\mu \mu}(\Delta x)$

$$
r_{\mu \mu}(\Delta x)=e^{-|\Delta x| / D}
$$

where $D$ is called the decorrelation distance, which is an area-dependent real-valued constant (see Table II). From (12) it can be seen that the spatial ACF $\hat{r}_{\mu \mu}(\Delta x)$ of the shadowing simulator is a function of the gains $c_{n}$, the discrete spatial frequencies $\alpha_{n}$, and the number of sinusoids $N$. Figures 2 and 3 show that the ACFs $\hat{r}_{\mu \mu}(\Delta x)$ of the measurement-based simulation model fit very well to the measured spatial ACFs $r_{\mu \mu}^{\star}(\Delta x)$. The results for the Gudmundson correlation model were also presented by using the decorrelation distance $D$ given in Table II. Figures 4 and 5 show that the quality of the approximation of the spatial ACFs $\hat{r}_{\lambda \lambda}(\Delta x)$ [see (14)], achieved by applying the proposed measurement-based model, is much better than the approximation of the spatial ACFs $r_{\lambda \lambda}(\Delta x)$ [see (4)], provided by the Gudmundson model.

\subsection{Evaluation of the Decorrelation and the Coherence Distances}

The decorrelation distance $D$ of the Gudmundson model is defined as the value of $\Delta x$ for which the spatial ACF $r_{\mu \mu}(\Delta x)$ in (22) equals $1 / e$, i.e., $\left.r_{\mu \mu}(\Delta x)\right|_{\Delta x=D}=$ $1 / e$. The decorrelation distance $D^{\star}$ of the measured channel and the decorrelation distance $\hat{D}$ of the measurement-based simulation model have been determined and compared with $D$. The results for the suburban and urban area are presented in Table III. This table includes also the results for the coherence distance. The coherence distance $D_{c}$ of the Gudmundson model has been obtained by substituting (22) in (7). This allows us 


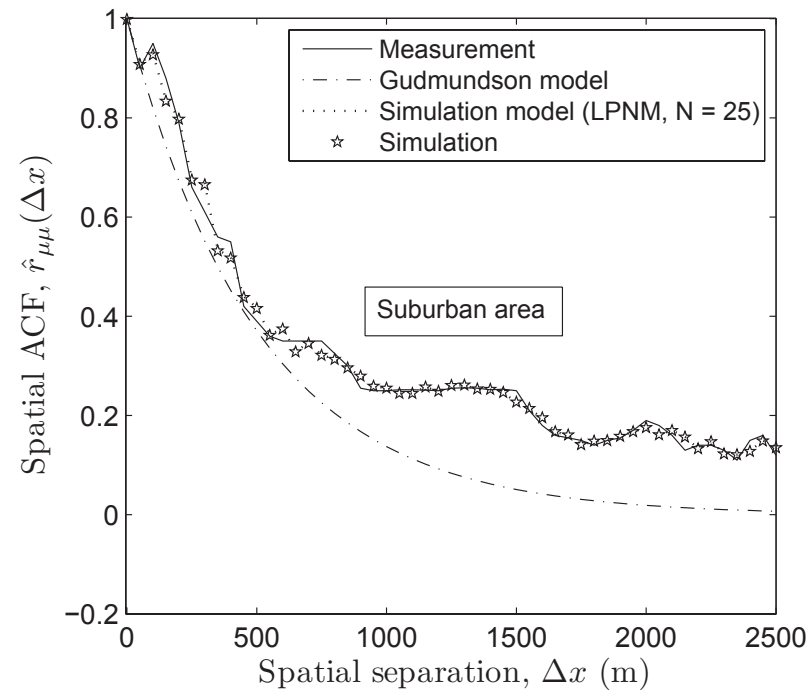

Figure 2. Spatial ACFs $r_{\mu \mu}^{\star}(\Delta x)$ (measured channel) in comparison with the spatial ACFs $r_{\mu \mu}(\Delta x)$ (Gudmundson model) and $\hat{r}_{\mu \mu}(\Delta x)$ (simulation model) for the suburban area.

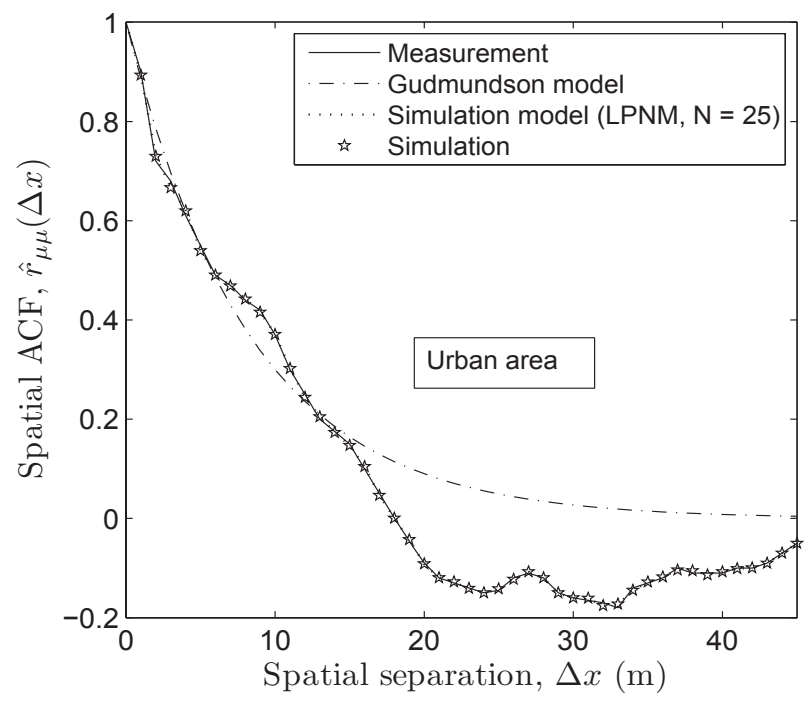

Figure 3. Spatial ACFs $r_{\mu \mu}^{\star}(\Delta x)$ (measured channel) in comparison with the spatial ACFs $r_{\mu \mu}(\Delta x)$ (Gudmundson model) and $\hat{r}_{\mu \mu}(\Delta x)$ (simulation model) for the urban area.

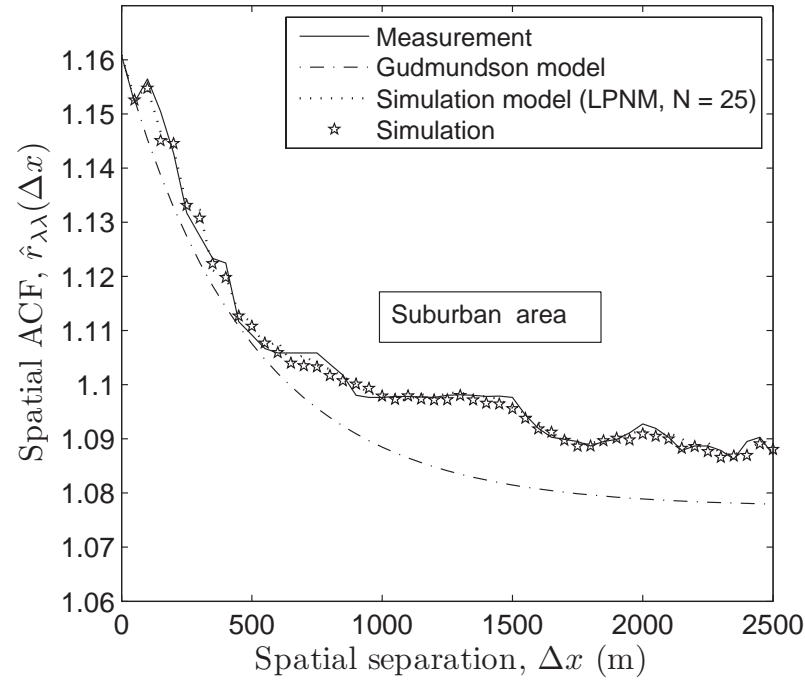

Figure 4. Spatial ACFs $r_{\lambda \lambda}^{\star}(\Delta x)$ (measured channel) in comparison with the spatial ACFs $r_{\lambda \lambda}(\Delta x)$ (Gudmundson model) and $\hat{r}_{\lambda \lambda}(\Delta x)$ (simulation model) for the suburban area.

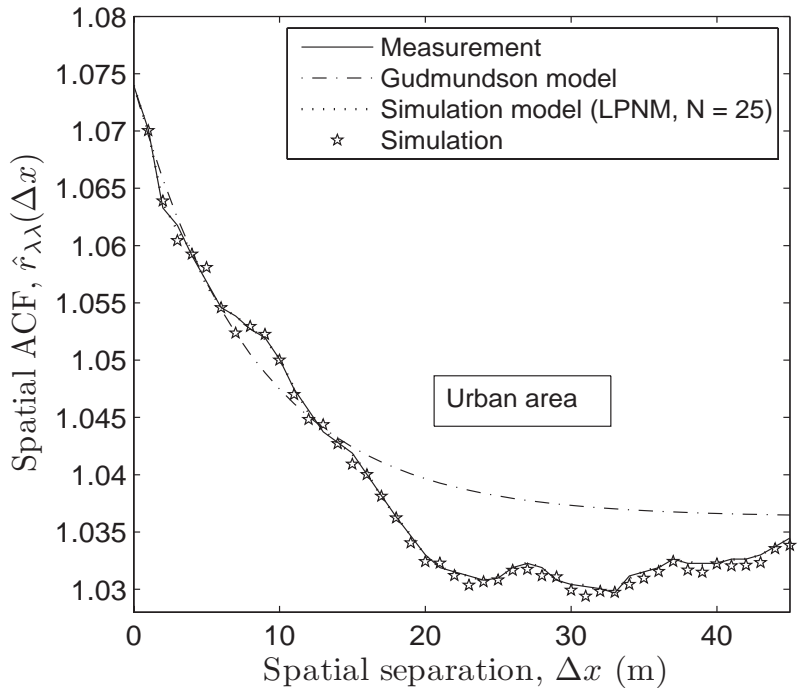

Figure 5. Spatial ACFs $r_{\lambda \lambda}^{\star}(\Delta x)$ (measured channel) in comparison with the spatial ACFs $r_{\lambda \lambda}(\Delta x)$ (Gudmundson model) and $\hat{r}_{\lambda \lambda}(\Delta x)$ (simulation model) for the urban area.

Table III

The Decorrelation and Coherence Distances

\begin{tabular}{|c||c|c|}
\hline $\begin{array}{c}\text { Decorrelation } \\
\text { and Coherence } \\
\text { Distances }\end{array}$ & Suburban area & Urban area \\
\hline \hline Measured channel: $D^{\star}$ & $525 \mathrm{~m}$ & $10 \mathrm{~m}$ \\
\hline Simulation model: $\hat{D}$ & $520.19 \mathrm{~m}$ & $10.39 \mathrm{~m}$ \\
\hline Gudmundson's model: $D$ & $503.9 \mathrm{~m}$ & $8.3058 \mathrm{~m}$ \\
\hline \hline Measured channel: $D_{c}^{\star}$ & $398 \mathrm{~m}$ & $5.79 \mathrm{~m}$ \\
\hline Simulation model: $\hat{D}_{c}$ & $397.3 \mathrm{~m}$ & $5.826 \mathrm{~m}$ \\
\hline Gudmundson's model: $D_{c}$ & $339.98 \mathrm{~m}$ & $5.683 \mathrm{~m}$ \\
\hline
\end{tabular}

to express $D_{c}$ in terms of $D$ as

$$
D_{c}=-D \ln \left(\frac{1}{\sigma_{0}^{2}} \ln \left(\frac{e^{\sigma_{0}^{2}}+1}{2}\right)\right)
$$

where $\sigma_{0}=\sigma_{L} \ln (10) / 20$. The coherence distance $\hat{D}_{c}$ of the measurement-based simulation model has been found by first substituting (15) in (16) and then solving numerically the obtained equation by using root finding techniques. Finally, the coherence distance $D_{c}^{\star}$ of the measured channel has been obtained from the graphs with solid lines in Figures 2 and 3. It is noticeable that the coherence distance $\hat{D}$ of the measurement-based simulation model is much closer to the coherence distance $D_{c}^{\star}$ of the measured channel than the coherence distance $D_{c}$ of the Gudmundson model.

\subsection{Evaluation of the LCR and the ADF}

A drawback of the Gudmundson model is that this correlation model results in an infinite LCR, because the second derivative of the spatial ACF $r_{\mu \mu}(\Delta x)$ in (22) is indefinite at the origin [15]. Figure 6 depicts the approximation results of the LCR $\hat{N}_{\lambda}(r)$ obtained by substituting the model parameters listed in Table I in (18). In addition, we have illustrated the exact solution of the LCR $\hat{N}_{\lambda}(r)$ by using the expression in [17, eq. (15)]. The simulation results confirm the correctness of the 
exact solution, whereas the approximate solution in (18) becomes less accurate at high signal levels. Figure 7 shows us the resulting graphs for the ADF $\hat{T}_{\lambda_{-}}(r)$ of the shadowing simulator, which have been obtained by evaluating (20) using the approximate [see (18)] as well as exact [see [17, eq. (15)]] solutions of the LCR. The simulation results fit very well with garaphs of the ADF $\hat{T}_{\lambda_{-}}(r)$, while using the exact solution of the LCR $\hat{N}_{\lambda}(r)$.

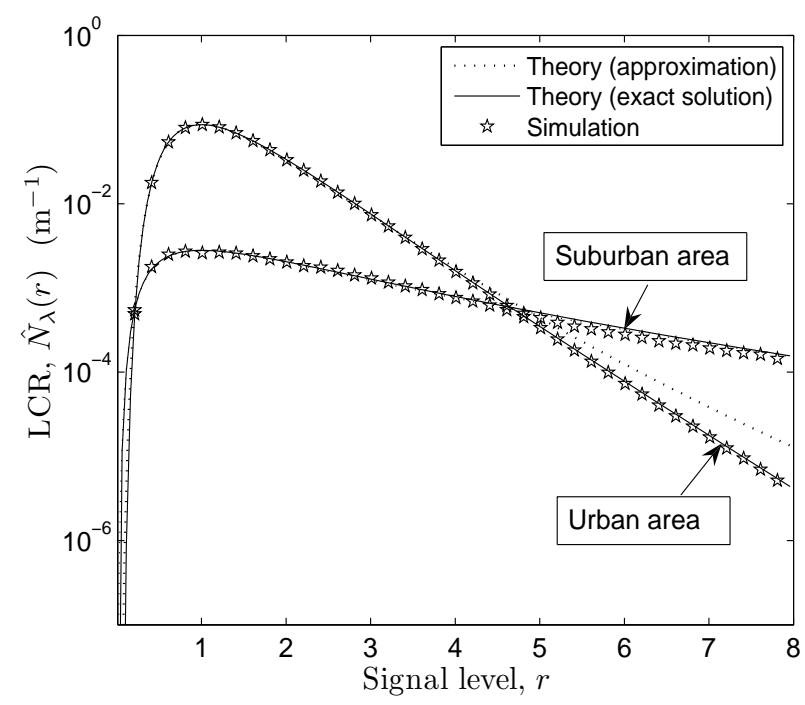

Figure 6. Comparison of the exact and approximate solutions for the LCR $\hat{N}_{\lambda}(r)$ of the measurement-based simulation model for suburban and urban areas $(N=25)$.

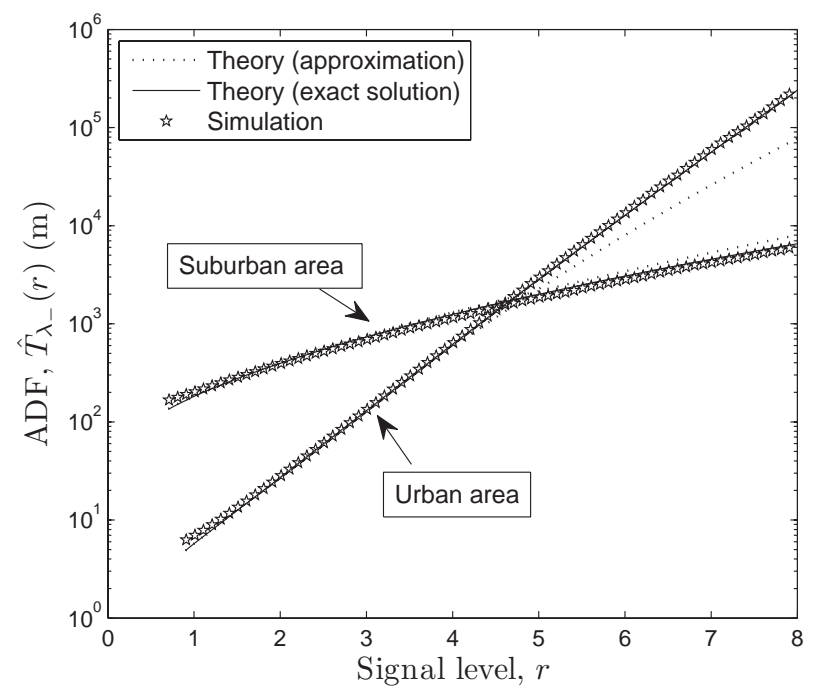

Figure 7. Comparison of the exact and approximate solutions for the ADF $\hat{T}_{\lambda_{-}}(r)$ of the measurement-based simulation model for suburban and urban areas $(N=25)$.

\section{Simulation Results}

In this section, we present some simulation results of the ergodic spatial shadowing processes designed for suburban and urban areas. From (10) and (11), we can infer the structure of the proposed shadowing simulator shown in Figure 8.
By using the model parameters $c_{n}$ and $\alpha_{n}$ listed in Table I along with the parameters $\sigma_{L}$ and $m_{L}$ in Table II, the simulation of the measurement-based spatial shadowing process can be performed. The resulting deterministic lognormal processes $\tilde{\lambda}^{(i)}(x)(i=1,2, \ldots)$ are obtained from the stochastic process $\hat{\lambda}(x)$ by replacing the random phases $\theta_{n}$ in (11) by constant quantities. By computing different sets $\left\{\theta_{1}^{(i)}, \theta_{2}^{(i)}, \ldots, \theta_{N}^{(i)}\right\}$, we can generate different deterministic lognormal processes $\tilde{\lambda}^{(i)}(x)$, all of them having the same statistics if the key parameters $\left(c_{n}, \alpha_{n}, \sigma_{L}\right.$, and $\left.m_{L}\right)$ are kept constant. Due to the ergodic properties of $\hat{\lambda}(x)$, all waveforms $\tilde{\lambda}^{(1)}(x), \tilde{\lambda}^{(2)}(x), \ldots$ have the same statistical properties. The fading behavior of shadowing in suburban and urban areas is shown in Figures 9 and 10, respectively. Taking into account the different scaled $x$-axes, one can realize that for suburban areas, the sample function $\tilde{\lambda}^{(i)}(x)$ fluctuates very slowly compared to the fluctuations in urban areas.

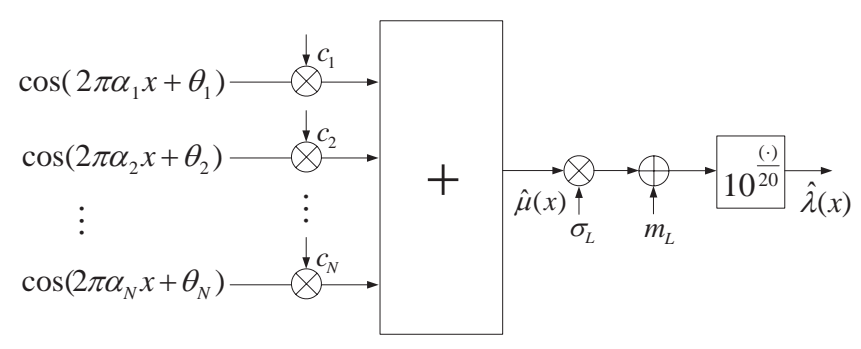

Figure 8. Structure of the shadowing simulator.

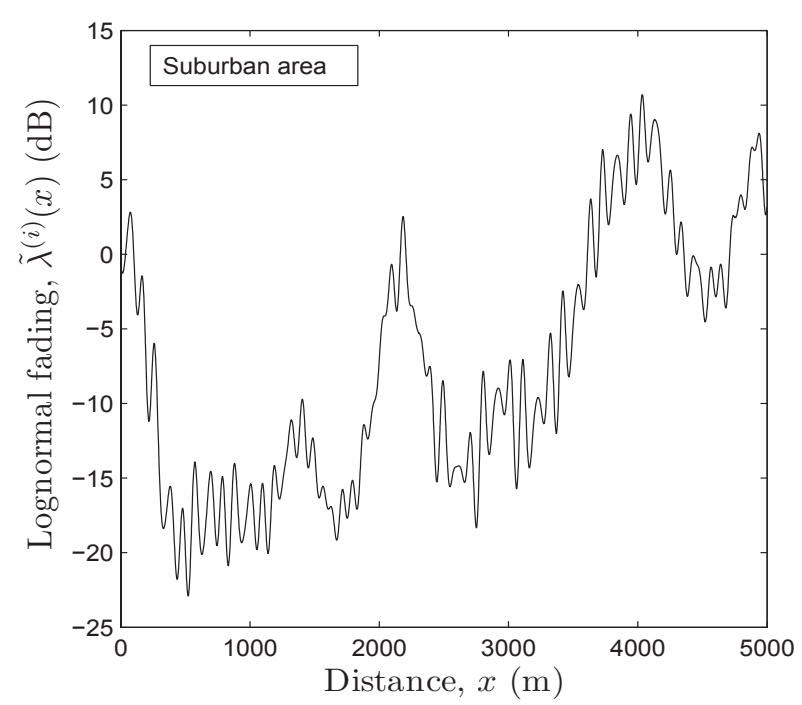

Figure 9. Simulation of the measurement-based spatial shadowing process $\tilde{\lambda}^{(i)}(x)$ for a suburban area $(N=25)$.

\section{Conclusion}

In this paper, a procedure for the design of measurement-based spatial correlation models for shadow fading has been proposed. The proposed procedure is based on the SOS principle. The LPNM has been used to compute the model parameters of 


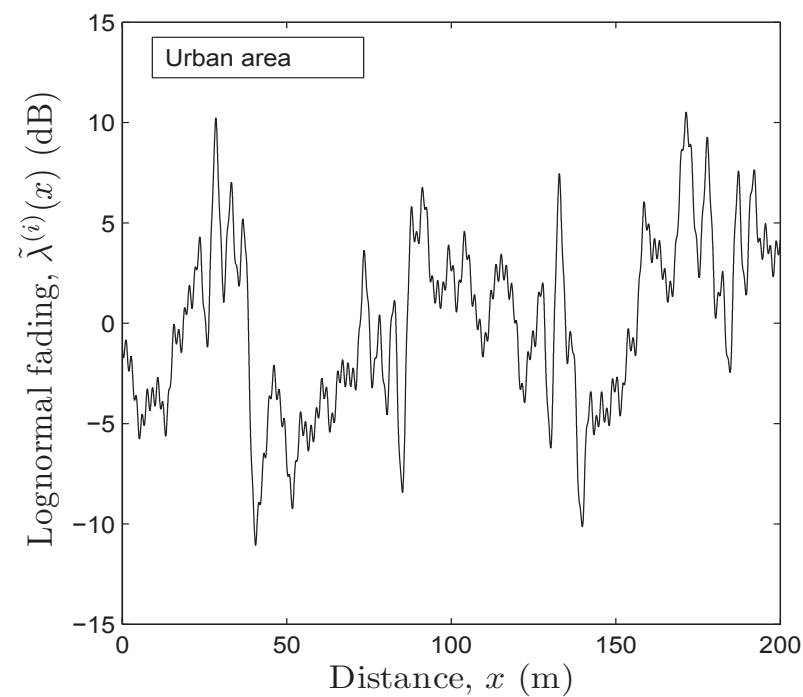

Figure 10. Simulation of the measurement-based spatial shadowing process $\tilde{\lambda}^{(i)}(x)$ for an urban area $(N=25)$.

the SOS by fitting the spatial ACF of the shadowing simulator to that of a measured channel. The design procedure has been applied to real-world measurement data collected in suburban and urban areas. The correlation properties of the proposed shadowing simulator have been compared with those of the Gudmundson model. A comparison has also been made with respect to the goodness of fitting of the spatial ACF of the shadowing simulator to that of a measured channel. Apart from the spatial ACF, we have also studied analytically the decorrelation and coherence distance as well as the LCR and the ADF. The correctness of all analytical results has been confirmed by simulations, which demonstrated that the proposed measurementbased correlation model provides an excellent fitting to real-world channels and outperforms the Gudmundson correlation model by far.

\section{REFERENCES}

[1] Y. Okumura, E. Ohmori, T. Kawano, and K. Fukuda, "Field strength and its variability in VHF and UHF land mobile radio services," Review of the Elec. Comm. Lab, vol. 16, pp. 825-873, Sept./Oct. 1968.

[2] D. O. Reudink, "Comparison of radio transmission at $\mathrm{X}$-band frequencies in suburban and urban areas," IEEE Transactions on Antennas and Propagation, vol. 20, pp. 470473, Jul. 1972.

[3] D. M. Black and D. O. Reudnik, "Some characteristics of mobile radio propagation at $836 \mathrm{MHz}$ in the Philadelphia area," IEEE Transactions on Vehicular Technology, vol. 21, pp. 45-51, May 1972.

[4] M. F. Ibrahim and J. D. Parsons, "Signal strength prediction in built-up areas," Proceedings of the IEE, vol. 130F, no. 5, pp. 377-384, 1983.

[5] H. Suzuki, "A statistical model for urban radio propagation," IEEE Transactions on Communications, vol. 25, no. 7, pp. 673-680, Jul. 1977.

[6] C. Loo and J. S. Butterworth, "Land mobile satellite channel measurements and modeling," Proceedings of the IEEE, vol. 86, no. 7, pp. 1442-1463, Jul. 1998.

[7] M. Pätzold, Y. Li, and F. Laue, "A study of a land mobile satellite channel model with asymmetrical Doppler power spectrum and lognormal distributed line-of-sight component," IEEE Transactions on Vehicular Technology, vol. 47, no. 1, pp. 297-310, Feb. 1998.

[8] A. K. Papazafeiropoulos and S. A. Kotsopoulos, "An extended Loo model with inhomogeneous scattering and its statistical properties," Wireless Personal Communications, vol. 52, no. 2, pp. 163-180, Oct. 2009.

[9] W. Lee and D. H. Cho, "A new velocity estimation scheme based on spatial correlation of wireless communication channel," in Proc. IEEE Wireless Communications and Networking Conference (WCNC), Budapest, Hungary, 2009, pp. 2208-2213.

[10] — , "Mean velocity estimation of mobile stations by spatial correlation of channels in cellular systems," IEEE Communications Letters, vol. 13, no. 9, pp. 670-672, Sept. 2009.

[11] K. Yamamoto, A. Kusuda, and S. Yoshida, "Impact of shadowing correlation on coverage of multihop cellular systems," in Proc. IEEE Int. Conference on Communications (ICC), vol. 10, June 2006, pp. 4538-4542.

[12] G. P. Pollini, "Trends in handover design," IEEE Communications Magazine, vol. 34, no. 3, pp. 82-90, Mar. 1996.

[13] N. Zhang and J. M. Holtzman, "Analysis of handoff algorithms using both absolute and relative measurements," IEEE Transactions on Vehicular Technology, vol. 45, no. 1, pp. 174-179, Feb. 1996.

[14] M. Gudmundson, "Correlation model for shadow fading in mobile radio systems," Electronics Letters, vol. 27, no. 23, pp. 2145-2146, Nov. 1991.

[15] D. Giancristofaro, "Correlation model for shadow fading in mobile radio channels," Electronics Letters, vol. 32, no. 11, pp. 958-959, May 1996.

[16] M. Pätzold and V. Nguyen, "A spatial simulation model for shadow fading processes in mobile radio channels," in Proc. 19th IEEE Int. Symposium on Personal, Indoor and Mobile Radio Communications (PIMRC), vol. 3, Barcelona, Spain, Sept. 2004, pp. 1832-1838.

[17] M. Pätzold and K. Yang, "An exact solution for the levelcrossing rate of shadow fading processes modelled by using the sum-of-sinusoids principle," in Proc. 9th Int. Symp. WPMC, San Diego, CA, Sept. 2006, pp. 188-193.

[18] A. Hong, M. Narandzic, C. Schneider, and R. Thomä, "Estimation of the correlation properties of large scale parameters from measurement data," in Proc. 18th IEEE Int. Symposium on Personal, Indoor and Mobile Radio Communications (PIMRC), Athens, Greek, Sept. 3-7 2007, pp. $1-5$.

[19] P. Agrawal and N. Patwari, "Correlated link shadow fading in multi-hop wireless networks," IEEE Transactions on Wireless Communications, vol. 8, no. 8, pp. 4024-4036, Aug. 2009.

[20] J. Poutanen, K. Haneda, V. M. Kolmonen, J. Salmi, and P. Vainikainen, "Analysis of correlated shadow fading in dual-link indoor radio wave propagation," IEEE Antennas and Wireless Propagation Letters, vol. 8, pp. 1190-1193, 2009.

[21] Y. Zhang, J. Zhang, D. Dong, X. Nie, G. Liu, and P. Zhang, "A novel spatial autocorrelation model for shadow fading in urban macro environments," in Proc. IEEE Global Telecommunications Conference (GLOBECOM), Nov. 30-Dec. 4 2008, pp. 1-5.

[22] M. Pätzold and B. O. Hogstad, "Classes of sum-ofsinusoids Rayleigh fading channel simulators and their stationary and ergodic properties - Part I," WSEAS Transactions on Mathematics, vol. 5, no. 1, pp. 222-230, Feb. 2006.

[23] M. Pätzold, Mobile Fading Channels. Chichester: John Wiley \& Sons, 2002. 


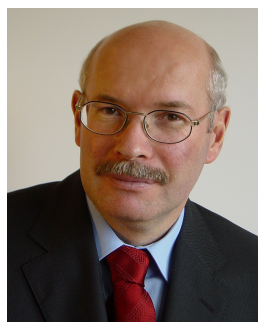

Matthias Pätzold received the Dipl.-Ing. and Dr.-Ing. degrees in Electrical Engineering from Ruhr-University Bochum, Bochum, Germany, in 1985 and 1989, respectively, and the habil. degree in Communications Engineering from the Technical University of HamburgHarburg, Hamburg, Germany, in 1998. From 1990 to 1992, he was with ANT Nachrichtentechnik GmbH, Backnang, Germany, where he was engaged in digital satellite communications. From 1992 to 2001, he was with the department of digital networks at the Technical University HamburgHarburg. Since 2001, he has been a full professor of mobile communications with the University of Agder (UiA), Grimstad, Norway. He authored several books andmore than 200 technical papers. His publications received eight best paper awards. He has been actively participating in numerous conferences serving as TPC chair and TPC member.

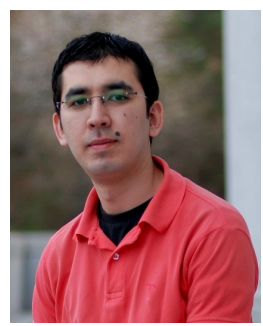

Nurilla Avazov was born in Tashkent, Uzbekistan. He received his BSc and MSc degrees in Telecommunications from Tashkent University of Information Technologies, Tashkent, Uzbekistan, in 2005 and 2007, respectively. In 2007, the Korean Government awarded him a scholarship granted by the Institute of Information Technology Advancement (IITA) for a research assistantship at the Kyung Hee University, School of Electronics and Information, Korea.

Since 2009, he has been working on his $\mathrm{PhD}$ thesis under supervision of Professor Matthias Pätzold at the University of Agder (UiA), Grimstad, Norway. His current research interests include MIMO systems and channel modelling for mobile-to-mobile communication systems.

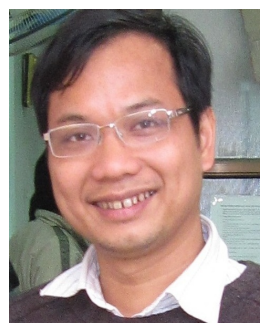

Van Duc Nguyen was born in Vietnam, in 1973. He received the Bachelor and Master of Engineering degrees in Electronics and Communications from the Hanoi University of Science and Technology (HUST), Vietnam, in 1995 and 1997, respectively, and the Dr.Eng. degree in Communications Engineering from the University of Hannover, Germany, in 2003.

From 1995 to 1998, he worked for the HUST as an assistant researcher. In 1996, he participated in the student exchange program between the HUST and the Munich University of Applied Sciences for one term. From 1998 to 2003, he was with the Institute of Communications Engineering, University of Hannover, first as a DAAD scholarship holder and then as a member of the scientific staff. From 2003 to 2004, he was employed with Agder University College in Grimstad, Norway, as a postdoctoral researcher. He was with the International University of Bremen as a postdoctoral fellow. In 2007, he spent 2 months at the Sungkyungkwan University, Korea, as a research professor. In 2008 and 2009, he was a visiting researcher at the Klagenfurt University, Austria, and at the University of Agder, Norway, respectively. His current research interests include mobile radio communications, especially MIMO-OFDM technology for 3GPP LTE networks. 